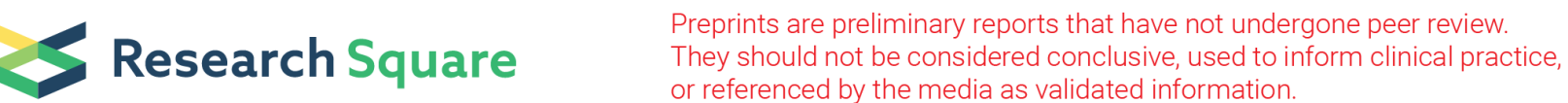

\section{Variations in Hybrid Combinations Lead to Differences in Mitochondrial Inheritance}

\section{Liming Luo}

Guangxi Academy of Fishery Sciences https://orcid.org/0000-0003-2459-2576

\section{Qiting Zou}

Guangxi Academy of Fishery Sciences

Junneng Liang

Guangxi Academy of Fishery Sciences

\section{Yongju Luo}

Guangxi Academy of Fishery Sciences

Qinglang Yin

Guangxi Academy of Fishery Sciences

\section{Liuqing Li}

Guangxi Academy of Fishery Sciences

\section{Xiaojin Zhang}

Guangxi Academy of Fishery Sciences

\section{Wufang Tan}

Guangxi Academy of Fishery Sciences

\section{Qiyu Wei}

Guangxi Academy of Fishery Sciences

\section{Zhuojun Han}

Guangxi Academy of Fishery Sciences

\section{Jingyi Zhang}

Guangxi Academy of Fishery Sciences

Jingjie Wang

Guangxi Academy of Fishery Sciences

\section{Xu Zhang}

Guangxi Academy of Fishery Sciences

Jun Xiao ( $\nabla$ dreamshaw@foxmail.com )

Guangxi Academy of Fishery Sciences https://orcid.org/0000-0001-6559-7651

\section{Research article}

Keywords: Hybrid tilapia, Mitochondria DNA, Maternal Inheritance, Paternal Inheritance. 
Posted Date: January 19th, 2021

DOI: https://doi.org/10.21203/rs.3.rs-147922/v1

License: (c) (i) This work is licensed under a Creative Commons Attribution 4.0 International License. Read Full License 


\section{Abstract}

\section{Background}

While tilapia are the second most farmed group of fish in the world, the Oreochromis niloticus $(\mathbb{\nabla}) \times$ Oreochromis aureus $(\mathbb{Z})$ hybrid is one of the most frequently observed tilapia crosses in China. Based on its conservative nature and maternal inheritance pattern, mitochondrial DNA is often used in kinship analysis. Evidence of paternal inheritance has been noted in some animal species.

\section{Results}

The mitochondrial Col and Cytb genes, and D-loop gene regions of Oreochromis niloticus and Oreochromis aureus fish were sequenced and aligned to their orthogonal and backcrossed offspring. As evidence of paternal mitochondria DNA inheritance was found, the whole mitochondrial genome was then sequenced. Results showed that in the Oreochromis niloticus $(\mathbb{\nabla}) \times$ Oreochromis aureus $(\mathbb{\nabla})$ hybrids, certain fish shared $92.88 \%$ of the maternal mitochondria genome, and $99.86 \%$ with the paternal mitochondria genome. This implied that there was paternal mtDNA inheritance. However, all Oreochromis niloticus $(\mathbb{\nabla}) \times$ Oreochromis aureus $(\mathbb{\nabla})$ hybrids had $100 \%$ identical mitochondria genome with their female parent.

\section{Conclusions}

The study showed that while paternal mtDNA inheritance occurred in the Oreochromis niloticus $(\mathbb{\nabla}) \times$ Oreochromis aureus $(\mathbb{\nabla})$ hybrid, this did not happen in Oreochromis niloticus $(\mathbb{\nabla}) \times$ Oreochromis aureus $(\mathbb{\nabla})$ offspring. This implies that in hybrid species, different hybridization combinations might provide an explanation for paternal mtDNA inheritance pattern.

\section{Background}

Present in the mitochondria of most eukaryotic cells, mitochondrial (mt)DNA is a small, circular, conserved double-chained DNA molecule when compared to nuclear DNA. While the mtDNA can selfreplicate and translate, it is also under the control of the nuclear genome. Animal mtDNA is $15-20 \mathrm{~kb}$ in length, and houses 13 protein coding genes, two rRNA and 22 tRNA genes. The order in which genes are found is stable [1, 2]. Generally, maternal mtDNA is inherited by offspring $[3,4]$ in most eukaryotes in whom sexual reproduction takes place. Since paternal mtDNA is reduced during spermatogenesis or eliminated after fertilization $[5,6], \mathrm{mtDNA}$ is one of the most stable and useful tools in population genetics and kinship analysis [3]. The theory that humans originated from Africa [7] is one example of mtDNA analysis results.

However, studies have found several species, such as Mytilus galloprovincialis [8] and Modiolus modiolus [9], Meloidogyne javanica [10], humans [11, 12], Drosophila [13] and triploid crucian carp [14], 
with evidence supporting the paternal inheritance of mtDNA. Do these evidences threaten the correctness in the theory of maternal mtDNA inheritance?

Tilapia are the second most farmed group of fish in the world [15] due to their high economic value and environmental adaptability [2]. Belonging to the same genera, male Nile tilapia (Oreochromis niloticus) and female Blue tilapia (Oreochromis aureus) are used by farmers to raise hybrids in China because it produces a predominantly male population (more than $95 \%$ of fish are male) that has superior growth when compared to females [16]. Additionally, while the orthogonal hybrid tilapia have a higher growth rate than either Nile or Blue tilapia, the male-dominated hybrid population helps reduce unwanted reproduction that could cause overpopulation and malnutrition. The high-male population of orthogonal hybrid tilapia is considered the consequence of different types of sex determination systems between Nile (XX/XY) and Blue tilapia (ZW/ZZ) [16-18]. Because of this, orthogonal hybrid tilapia result in male fish that have a stable pair of sex chromosomes. However, unlike the orthogonal hybrid tilapia, the backcrossing of tilapia results in a normal sex ratio of $50 \%$ male and $50 \%$ female. The difference in sex chromosome pairing makes the backcrossed hybrid tilapia a better experimental animal in the study of hybrid tilapia.

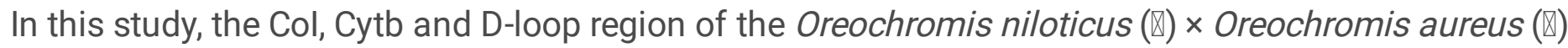

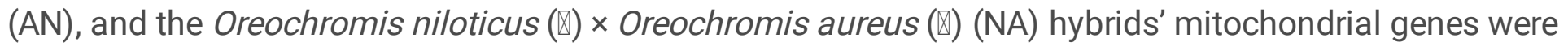
sequenced. Evidence of paternal mtDNA inheritance was found. The whole mt genomes of hybrid AN and NA tilapia were subsequently sequenced and aligned against the sequences obtained from their Oreochromis niloticus and Oreochromis aureus parents. By analyzing the data, it was observed that paternal mtDNA transfer occurred in the hybrid AN tilapia, while maternal mtDNA was inherited in the NA hybrid tilapia. This result may provide new insights into mtDNA inheritance in different populations crossing.

\section{Results}

\section{The difference in appearances}

Images of the experimental Nile tilapia, Blue tilapia, orthogonal hybrid tilapia (female Nile tilapia $\times$ male Blue tilapia) and backcrossed hybrid tilapia (male Nile tilapia $\times$ female Blue tilapia) are shown in Fig. 1. Nile tilapia have a striped caudal fin pattern with no spots on the operculum. Blue tilapia have dotted caudal fin patterns and spots on the operculum. The orthogonal and backcrossed hybrid tilapia had the same appearance that combined the characteristics of their male and female parents. This included striped but light caudal fin patterning and colored spots on the operculum.

\section{Col gene}

The mt Col gene length was 1,595 bp in all four experimental tilapia groups. The similarity in Col genes between the parent Nile and Blue tilapia was $93.17 \%$, proving that the Col gene in Nile and Blue tilapia share the same coding sequence but have different codons. The orthogonal hybrid AN17 and AN18 
tilapia shared $100 \%$ identity with their female parent Nile tilapia, suggesting the Col gene in AN17/AN18 and NA are maternally inherited. Moreover, the Col gene of orthogonal AN3 and AN4 hybrid tilapia both shared $93.17 \%$ identity with their female Nile tilapia parent, and $100 \%$ identity with their male Blue tilapia parent. This suggested that paternal $\mathrm{mt}$ Col gene leakage occurred in orthogonal AN3/AN4 hybrid tilapia. Contrarily, the Col gene of backcrossed hybrid NA tilapia shared $100 \%$ identity with their female Blue tilapia parent, suggesting that the Col gene in backcrossed hybrid NA tilapia strictly followed the maternal inheritance pattern.

\section{Cytb gene}

The $\mathrm{mt}$ Cytb gene that was sequenced in parental and offspring tilapia was $1,140 \mathrm{bp}$ in length. Showing the difference between the male and female parent tilapia, the similarity between the Cytb gene across the parent Nile and Blue tilapia fish was $89.66 \%$. The AN17 and AN18 mt Cytb gene shared $100 \%$ identity with its female Blue tilapia parent. However, the AN3 and AN4 Cytb gene both share $89.83 \%$ identity with their female Nile tilapia parent, and $99.82 \%$ identity with their male Blue tilapia parent. Since the paternal identity is significantly higher than the maternal identity, it is suggested that the paternal mt Cytb gene was inherited by the orthogonal hybrid AN3/AN4 tilapia. The results also showed that the mt Cytb gene of the backcrossed hybrid NA tilapia shared $100 \%$ identity with its female Blue tilapia parent. This suggests that paternal mtDNA inheritance did not occur in NA fish.

\section{D-loop region}

The $\mathrm{mt}$ D-loop region obtained from all four experimental tilapia groups showed variability in gene lengths. The similarity across the D-loop region of both the Nile and Blue parent tilapia was $92.66 \%$. AN17, AN18 and their female Nile tilapia parent shared a similar D-loop region length of around $540 \mathrm{bp}$, while AN3, AN4 and NA showed similarity to Blue tilapia, with a length of approximately $530 \mathrm{bp}$. The Blast analysis showed $100 \%$ identity of AN17/AN18 with their female parent Nile tilapia, while AN3/AN4 shared $92.47 \%$ identity with their female Blue tilapia parent, and $99.82 \%$ identity with their male Nile tilapia parent. This suggests that paternal mtDNA was inherited in orthogonal AN3/AN4 hybrid tilapia. However, the analysis results showed that NA has a high identity with its female Blue tilapia parent (99.82\%), and low identity with its male Nile tilapia parent (92.47\%), suggesting that the D-loop region of NA was inherited from their female Blue tilapia parent with no sign of paternal mtDNA inheritance.

Nucleotide identities: From the Blast results in orthogonal AN3/AN4 hybrid tilapia, the paternally inherited Col, Cytb and D-loop region genes covered over 11,000 bp of the whole mt genome. Whether the remaining $\mathrm{mt}$ genome is inherited paternally or maternally is still unclear. To verify whether the leakage of the paternal $\mathrm{mt}$ genes was the result of paternal and maternal mtDNA recombination, the whole $\mathrm{mt}$ genome of the orthogonal AN and backcrossed NA hybrid tilapia were aligned against both of their male and female parents. The results are shown in Tables 1 and 2. The mt genomes of Nile and Blue tilapia was $92.88 \%$ identical. The mt genomes of orthogonal AN3 and AN4 hybrid tilapia were closer to their male Blue tilapia parent $(99.86 \%)$ than their female Nile tilapia parent $(92.88 \%)$, while AN17 and AN18 had the same mt genome as the female Nile tilapia parent (100\%). This suggested that the paternal 
mtDNA was included in the mt genome of AN3/AN4 hybrids, while AN17/AN18 hybrids inherited the whole maternal mt genome. In addition, in the whole AN3/AN4 mt genome, the separate gene nucleotide identity between the hybrid AN3/AN4 tilapia and its male Blue tilapia parent was higher than or equal to that between the hybrid AN3/AN4 tilapia and its female Nile tilapia parent. This indicated that in hybrid AN3/AN4 tilapia, instead of the recombination of paternal with maternal mtDNA, the whole paternal $\mathrm{mt}$ genome was inherited. The slight difference $(0.14 \%)$ between the $\mathrm{mt}$ genome of AN3/AN4 with their male Blue tilapia parent was considered the result of a point mutation in the paternal mtDNA that occurred during fertilization.

While paternal mtDNA inheritance happened in orthogonal hybrid AN tilapia, the complete mtDNA nucleotide identity of the backcrossed hybrid NA tilapia was found to be $100 \%$ identical to their female Blue tilapia parent and $94.16 \%$ identical with their male Nile tilapia parent. All sequenced $\mathrm{mt}$ Col, Cytb and D-loop region genes shared more similarity with the female Blue tilapia parent, suggesting that the mtDNA of hybrid NA tilapia were maternally inherited, and paternal mtDNA was reduced or eliminated as expected.

\section{Phylogenetic tree analysis}

We obtained the complete mtDNA sequences of zebrafish (NC_002333.2), Japanese medaka (NC_004387.1) and crucian carp (NC_006291.1) from GenBank while constructing the phylogenetic tree

with the complete Nile tilapia, Blue tilapia, orthogonal hybrid AN tilapia and backcrossed hybrid NA tilapia mtDNA sequences. The results are displayed in Fig. 2. As shown, AN3/AN4 tilapia were closely related to their male Blue tilapia parent, implying that the AN3/AN4 mtDNA was paternally inherited. Moreover, AN17/AN18 was genetically close to their female Nile tilapia parent, while NA was close to their female Blue tilapia parent. This suggested that their mtDNA was maternally inherited. The phylogenetic tree supported the previous results that paternal mtDNA inheritance happened randomly in AN, but not NA, fish.

\section{Discussion}

In this paper, and using the $\mathrm{mt}$ Col, Cytb genes and D-loop gene region as markers, significant evidence of

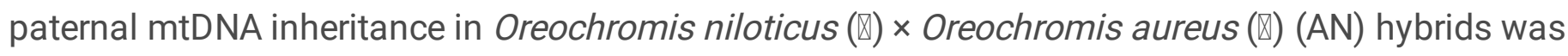

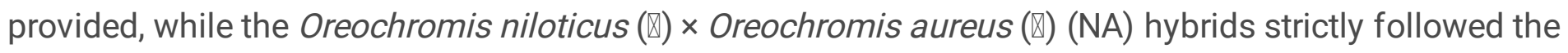
maternal mtDNA inheritance pattern. Blast analysis of these three markers revealed high levels of homology between orthogonal hybrid AN3/AN4 tilapia and their male Blue tilapia parent, and low levels of homology with their female Nile tilapia parent. NA tilapia had complete homology with the maternal mtDNA. The results indicated that paternal mtDNA leakage and inheritance occurred in the Oreochromis

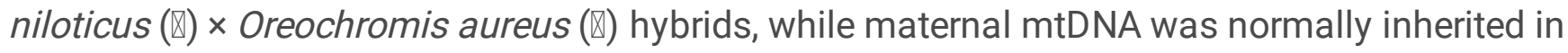

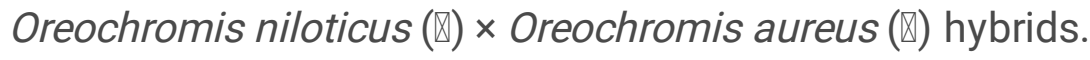

As paternal mitochondria are the energy supplying organelle in sperm, paternal mtDNA inheritance rarely happens during fertilization. By constantly generating energy, paternal mtDNA can easily mutate. 
Paternal mtDNA therefore needs to be eliminated, otherwise it can cause abnormal and defective embryos [19], mitochondrial myopathy [11] or sperm dysfunction [20]. There are different mechanisms that can eliminate paternal mtDNA in animals. These include ubiquitin-mediated degradation, fertilization-triggered autophagy, and paternal mtDNA degradation prior to the destruction of the mitochondrial structure [21]. For example, in Caenorhabditis elegans [22], paternal mitochondria entered the zygote at fertilization, and were fully eliminated after the ubiquitination of paternal membranous organelles by autophagy. Also, the mitochondrial endonuclease G CPS- 6 is considered an important paternal mitochondrial degradation factor during fertilization in C. elegans [23]. In Drosophila [5], the Tamas mtDNA polymerase showed its function in paternal mtDNA degradation during spermatogenesis.

In nature, paternal mtDNA inheritance mostly happened in bivalvian mollusks, like mussels [24, 25], due to the unique, doubly uniparental inheritance phenomenon in bivalvian mollusks [26, 27]. In these cases, the inherited paternal mtDNA only exist in the gonads and sperm of male mussels [28]. However, in previous studies, researchers found that paternal mtDNA inheritance in vertebrates was more common in interspecific crosses than in intraspecific crosses, with the probability of paternal mtDNA inheritance increasing with the increased phylogenetic distance of hybridizing species [29]. Paternal mtDNA inheritance in interspecific crosses, like in mice [30], sheep [31, 32], flatfish [33] and crucian carp [14, 34], usually happen when parts of paternal mtDNA recombine with maternal mtDNA.

This comparative study on nucleotide identities of whole $\mathrm{mt}$ genomes revealed that in hybrid AN tilapia, certain individuals shared a higher mtDNA identity with their male Blue tilapia parent $(98.66 \%$ average in AN3/AN4) than their female Nile tilapia parent (92.84\% average in AN3/AN4). Indicating the complete inheritance of paternal $\mathrm{mt}$ genome, AN tilapia were completely identical to their female Nile tilapia parent (100\% in AN17/AN18). In studying Oryzias latipes [35], researchers found that paternal mtDNA were reduced during spermatogenesis and then completely eliminated after fertilization. While the reason for the divergence in paternal and maternal mtDNA inheritance between orthogonal hybrid AN3/AN4 and AN17/AN18 tilapia is still unclear, it is hypothesized that this phenomenon is mainly cause by chromosomal synapsis errors in the nuclear genome. This leads to the dysfunction of the paternal mtDNA recognition or elimination system in the fertilized egg of orthogonal hybrid AN tilapia.

Theoretically, the low identity of maternal mtDNA could also be the result of mutations that occurred in hybrid AN tilapia. This would make the maternally inherited mtDNA of hybrid AN tilapia display a high nucleotide identity with its male parent. However, considering the proportion of differences between the female parent and the offspring, a large number of mutations are needed to shape the inherited mtDNA to be almost identical to the paternal mtDNA. Since mtDNA is highly conserved, such frequent point mutations are unlikely. We therefore conclude that in hybrid AN tilapia resulting from Oreochromis

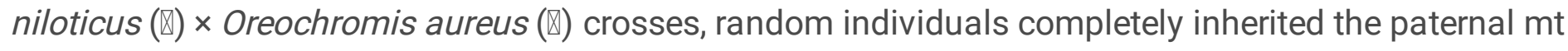
genome, while others inherited the maternal mtDNA.

The different mtDNA inheritance patterns between the orthogonal and backcrossed tilapia offspring is being reported for the first time. As the assumed invalid nuclear genes in the orthogonal hybrid AN tilapia 
were fully functional in the backcrossed hybrid NA tilapia, the paternal mtDNA in the fertilized eggs of NA fish can be eliminated. We hypothesize that the sex chromosome genes are responsible for the differences in mtDNA inheritance between orthogonal and backcrossed Oreochromis niloticus and Oreochromis aureus generations. This theory requires further investigation.

In summary, our study in tilapia show that in Oreochromis niloticus $\times$ Oreochromis aureus hybrids, different hybridization combinations could cause different mtDNA inheritance patterns. The orthogonal hybrid AN tilapia showed paternal mtDNA inheritance patterns, while the backcrossed hybrid NA tilapia showed complete maternal mtDNA inheritance. Our results may provide new insight into paternal mtDNA inheritance patterns.

\section{Conclusion}

In this paper, we demonstrate that in two different Oreochromis niloticus $\times$ Oreochromis aureus hybridizations, there are differences in mtDNA inheritance patterns. Our result suggests paternal mtDNA inheritance happened in certain hybrid combinations in interspecific crossed animals, implies different hybridization combinations may cause paternal mtDNA inheritance, adding a new perspective in mtDNA inheritance.

\section{Materials And Methods}

Animals: A permission were acquired to use the animals for experiment. Nile tilapia (N), Blue tilapia (A), and their orthogonal (AN) and backcrossed (NA) generations were bred at The National Tilapia Breeding Farm in Nanning, Guangxi, China. In March 2018, each experimental fish group was placed in a $1.5 \times 1.5$ $\times 1.5 \mathrm{~m}$ tank and paired at a ratio of $3 \rrbracket: 1 \rrbracket$. The breeding of four experimental groups of tilapia was finished in April 2018, and fish were allowed to continue breeding for four months. In October 2018, the caudal fin samples of four kinds of tilapia were collected and stored at $-80{ }^{\circ} \mathrm{C}$ for further use.

\section{DNA extraction and sequencing}

Total DNA was individually extracted from the caudal fin samples of 20 Nile tilapia, Blue tilapia, and the orthogonal and backcrossed generations, respectively. The DNA was extracted using the Ezup Column Animal Genomic DNA Purification Kit from Sangon Biotech Co., Ltd (Shanghai, China) according to the manufacturer's instructions. The complete mitochondria genome was sequenced by Sangon.

\section{Primers synthesis and PCR amplification}

Primer 5.0 was used to design three pairs of primers, as shown in Table 3. The primers were synthesized by Invitrogen Trading Co., Ltd. (Shanghai, China). The PCR reactions were conducted at $95{ }^{\circ} \mathrm{C}$ for 5 min and 34 cycles at $95^{\circ} \mathrm{C}$ for $40 \mathrm{~s}, 60^{\circ} \mathrm{C}$ for $60 \mathrm{~s}$ and $72{ }^{\circ} \mathrm{C}$ for $90 \mathrm{~s}$. 
Data analysis: PCR products were sequenced by Sangon, and all sequences were analyzed using Blast (http://www.ncbi.nlm.nih.gov) to determine their identity. A phylogenetic tree was constructed with MEGA$X[36]$, using the Neighbor-Joining method.

\section{Abbreviations}

$\mathrm{mt}$

Mitochondria

A

Oreochromis aureus

$\mathrm{N}$

Oreochromis niloticus

AN

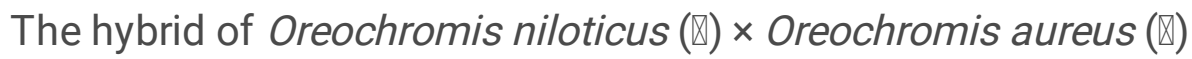

NA

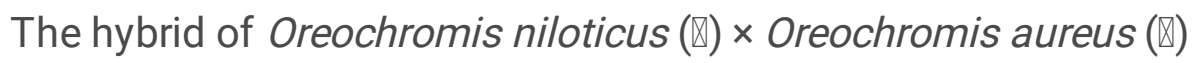

\section{Declarations}

\section{Availability of data and materials}

All mitochondrial genome data analyzed during the current study are available in the additional files.

\section{Acknowledgements}

No applicable.

\section{Funding}

The experimental materials and consumables for the experiments were supported by the National Natural Science Foundation of China [31560716]. The DNA sequencing were supported by the National Key Research and Development Program of China [2018YFD0900601] and the Natural Science Foundation of Guangxi [2016GXNSFFA380002]. The experimental animals and feed were supported by the China Agriculture Research System (CARS-46).

\section{Authors' information}

\section{Affiliations}

Guangxi Key Laboratory of Aquatic Genetic Breeding and Healthy Aquaculture, Guangxi Academic of Fishery Science, Nanning, 530021, China 
Liming Luo, Qiting Zou, Yongju Luo, Qinglang Yin, Liuqing Li, Xiaojin Zhang, Wufang Tan, Qiyu Wei, Zhuojun Han, Jingyi Zhang, Jingjie Wang, Xu Zhang, Jun Xiao and Junneng Liang.

Collage of Fishery and Life science, Shanghai Ocean University, Shanghai, 201306, China

Liming Luo, Yongju Luo, Qinglang Yin, Liuqing Li, Xiaojin Zhang, Wufang Tan, Qiyu Wei

College of Animal Science and Technology, Guangxi University, Nanning, 530004, China

Qiting Zou, Yongju Luo, Zhuojun Han, Jingyi Zhang, Jingjie Wang, Xu Zhang

\section{Authors Contributions}

To prevent confusion, we designate Liming Luo and Liuqing Li as LL1 and LL2, Xiaojin Zhang and Xu Zhang as ZX1 and ZX2, respectively. LL1, ZQ and XJ performed the data analysis and wrote the manuscript. ZQ, LY, XJ and LJ designed the study. ZQ, YQ, LL2, ZX1, TW, WQ, HZ, ZJ WJ and ZX2 conducted the experiments. $L Y, X J$ and $L J$ supervised the project. All authors read and approved the final manuscript. LL1, ZQ and LJ contributed equally to this work.

\section{Corresponding Authors}

Correspondence to Jun Xiao, Email: dreamshaw@foxmail.com

\section{Ethics declarations}

\section{Ethics approval and consent to participate}

All animal experiments were conducted according to the guidelines on the care and use of experimental animals established by the Ministry of Science and Technology of the People's Republic of China (Approval number: 2006-398) and was approved by the Guangxi Key Laboratory of Aquatic Genetic Breeding and Healthy Aquaculture, Guangxi Academic of Fishery Science.

\section{Consent for publication}

Not applicable.

\section{Competing interests}

The authors declare that they have no competing interests.

\section{References}

1. Ladoukakis ED, Zouros E. Evolution and inheritance of animal mitochondrial DNA: rules and exceptions. J Biol Res (Thessalon). 2017;24:2. 
2. He A, Luo Y, Yang H, Liu L, Li S, Wang C. Complete mitochondrial DNA sequences of the Nile tilapia (Oreochromis niloticus) and Blue tilapia (Oreochromis aureus): genome characterization and phylogeny applications. Mol Biol Rep. 2011;38(3):2015-21.

3. Yan J, Guo X, Liu S, Xiao J, Liu Z, Chen Y, Liu Y. Maternal inheritance in polyploid fish inferred from mitochondrial ATPase genes analysis. Prog Nat Sci. 2009;19(6):693-8.

4. Guo X, Liu S, Liu Y. Evidence for maternal inheritance of mitochondrial DNA in allotetraploid. DNA Seq. 2007;18(4):247-56.

5. Yu Z, O'Farrell PH, Yakubovich N, DeLuca SZ. The Mitochondrial DNA Polymerase Promotes Elimination of Paternal Mitochondrial Genomes. Curr Biol. 2017;27(7):1033-9.

6. Luo S-M, Ge Z-J, Wang Z-W, Jiang Z-Z, Wang Z-B, Ouyang Y-C, Hou Y, Schatten H, Sun Q-Y: Unique insights into maternal mitochondrial inheritance in mice. Proceedings of the National Academy of Sciences 2013, 110(32):13038.

7. Vigilant L, Stoneking M, Harpending H, Hawkes K, Wilson AC. African populations and the evolution of human mitochondrial DNA. Science. 1991;253(5027):1503.

8. Ladoukakis ED, Zouros E. Direct evidence for homologous recombination in mussel (Mytilus galloprovincialis) mitochondrial DNA. Mol Biol Evol. 2001;18(7):1168-75.

9. Robicheau BM, Breton S, Stewart DT. Sequence motifs associated with paternal transmission of mitochondrial DNA in the horse mussel, Modiolus modiolus (Bivalvia: Mytilidae). Gene. 2017;605:32-42.

10. Lunt DH, Hyman BC. Animal mitochondrial DNA recombination. Nature. 1997;387(6630):247.

11. Schwartz M, Vissing J. Paternal inheritance of mitochondrial DNA. N Engl J Med. 2002;347(8):57680.

12. Luo S, Valencia CA, Zhang J, Lee N-C, Slone J, Gui B, Wang X, Li Z, Dell S, Brown J, et al: Biparental Inheritance of Mitochondrial DNA in Humans. Proceedings of the National Academy of Sciences 2018, 115(51):13039.

13. Kondo R, Satta $\mathrm{Y}$, Matsuura E, Ishiwa H, Takahata N, Chigusa S. Incomplete Maternal Transmission of Mitochondrial DNA in Drosophila. Genetics. 1990;126:657-63.

14. Guo X, Liu S, Liu Y. Evidence for recombination of mitochondrial DNA in triploid crucian carp. Genetics. 2006;172(3):1745-9.

15. FAO. The state of world fisheries and aquaculture 2008. In.: FAO Rome; 2009.

16. Bartley DM, Rana K, Immink AJ. The use of inter-specific hybrids in aquaculture and fisheries. Rev Fish Biol Fisheries. 2000;10(3):325-37.

17. Baroiller JF, D'Cotta H, Bezault E, Wessels S, Hoerstgen-Schwark G. Tilapia sex determination: Where temperature and genetics meet. Comp Biochem Physiol A: Mol Integr Physiol. 2009;153(1):30-8.

18. Conte MA, Gammerdinger WJ, Bartie KL, Penman DJ, Kocher TD. A high quality assembly of the Nile Tilapia (Oreochromis niloticus) genome reveals the structure of two sex determination regions. BMC Genom. 2017;18(1):341. 
19. Chu Z, Guo W, Hu W, Mei J. Delayed elimination of paternal mtDNA in the interspecific hybrid of Pelteobagrus fulvidraco and Pelteobagrus vachelli during early embryogenesis. Gene. 2019;704:1-7.

20. Luo S-M, Schatten H, Sun Q-Y. Sperm Mitochondria in Reproduction: Good or Bad and Where Do They Go? Journal of Genetics Genomics. 2013;40(11):549-56.

21. Sato M, Sato K. Maternal inheritance of mitochondrial DNA by diverse mechanisms to eliminate paternal mitochondrial DNA. Biochim Biophys Acta. 2013;1833(8):1979-84.

22. Molina P, Lim Y, Boyd L. Ubiquitination is required for the initial removal of paternal organelles in C. elegans. Dev Biol. 2019;453(2):168-79.

23. Zhou Q, Li H, Li H, Nakagawa A, Lin JLJ, Lee E-S, Harry BL, Skeen-Gaar RR, Suehiro Y, William D, et al. Mitochondrial endonuclease $\mathrm{G}$ mediates breakdown of paternal mitochondria upon fertilization. Science. 2016;353(6297):394.

24. Liqin C. Differential segregation patterns of sperm mitochondria in embryos of the blue mussel (Mytilus edulis). Genetics 2004, 2(166).

25. Burzynski A, Zbawicka M, Skibinski DO, Wenne R. Evidence for recombination of mtDNA in the marine mussel Mytilus trossulus from the Baltic. Mol Biol Evol. 2003;20(3):388-92.

26. Zouros E. Biparental Inheritance Through Uniparental Transmission: The Doubly Uniparental Inheritance (DUI) of Mitochondrial DNA. Evol Biol. 2012;40(1):1-31.

27. Breton S, Beaupré HD, Stewart DT, Hoeh WR, Blier PU: The unusual system of doubly uniparental inheritance of mtDNA: isn't one enough? Trends in Genetics 2007, 23(9):465-474.

28. Sańko TJ, Burzyński A. Co-expressed mitochondrial genomes: recently masculinized, recombinant mitochondrial genome is co-expressed with the female - transmitted mtDNA genome in a male Mytilus trossulus mussel from the Baltic Sea. BMC Genet. 2014;15(1):28.

29. Dokianakis E, Ladoukakis ED. Different degree of paternal mtDNA leakage between male and female progeny in interspecific Drosophila crosses. Ecol Evol. 2014;4(13):2633-41.

30. Gyllensten U, Wharton D, Josefsson A, Wilson AC. Paternal inheritance of mitochondrial DNA in mice. Nature. 1991;352(6332):255-7.

31. Zhao X, Li N, Guo W, Hu X, Liu Z, Gong G, Wang A, Feng J, Wu C. Further evidence for paternal inheritance of mitochondrial DNA in the sheep (Ovis aries). Heredity. 2004;93(4):399-403.

32. Zhao X, Chu M, Li N, Wu C. Paternal inheritance of mitochondrial DNA in the sheep (Ovine aries). Science in China Series C: Life Sciences. 2001;44(3):321-6.

33. Hoarau G, Holla S, Lescasse R, Stam WT, Olsen JL. Heteroplasmy and evidence for recombination in the mitochondrial control region of the flatfish Platichthys flesus. Mol Biol Evol. 2002;19(12):2261-4.

34. Wang S, Jiao N, Zhao L, Zhang M, Zhou P, Huang X, Hu F, Yang C, Shu Y, Li W, et al. Evidence for the paternal mitochondrial DNA in the crucian carp-like fish lineage with hybrid origin. Science China Life Sciences. 2020;63(1):102-15.

35. Nishimura Y, Yoshinari T, Naruse K, Yamada T, Sumi K, Mitani H, Higashiyama T, Kuroiwa T. Active digestion of sperm mitochondrial DNA in single living sperm revealed by optical tweezers. Proc Natl 
Acad Sci U S A. 2006;103(5):1382-7.

36. Kumar S, Stecher G, Li M, Knyaz C, Tamura K. MEGA X: Molecular Evolutionary Genetics Analysis across Computing Platforms. Molecular biology and evolution 2018, 35.

\section{Tables}

Due to technical limitations, table 1 to 3 is only available as a download in the Supplemental Files section.

Figures 

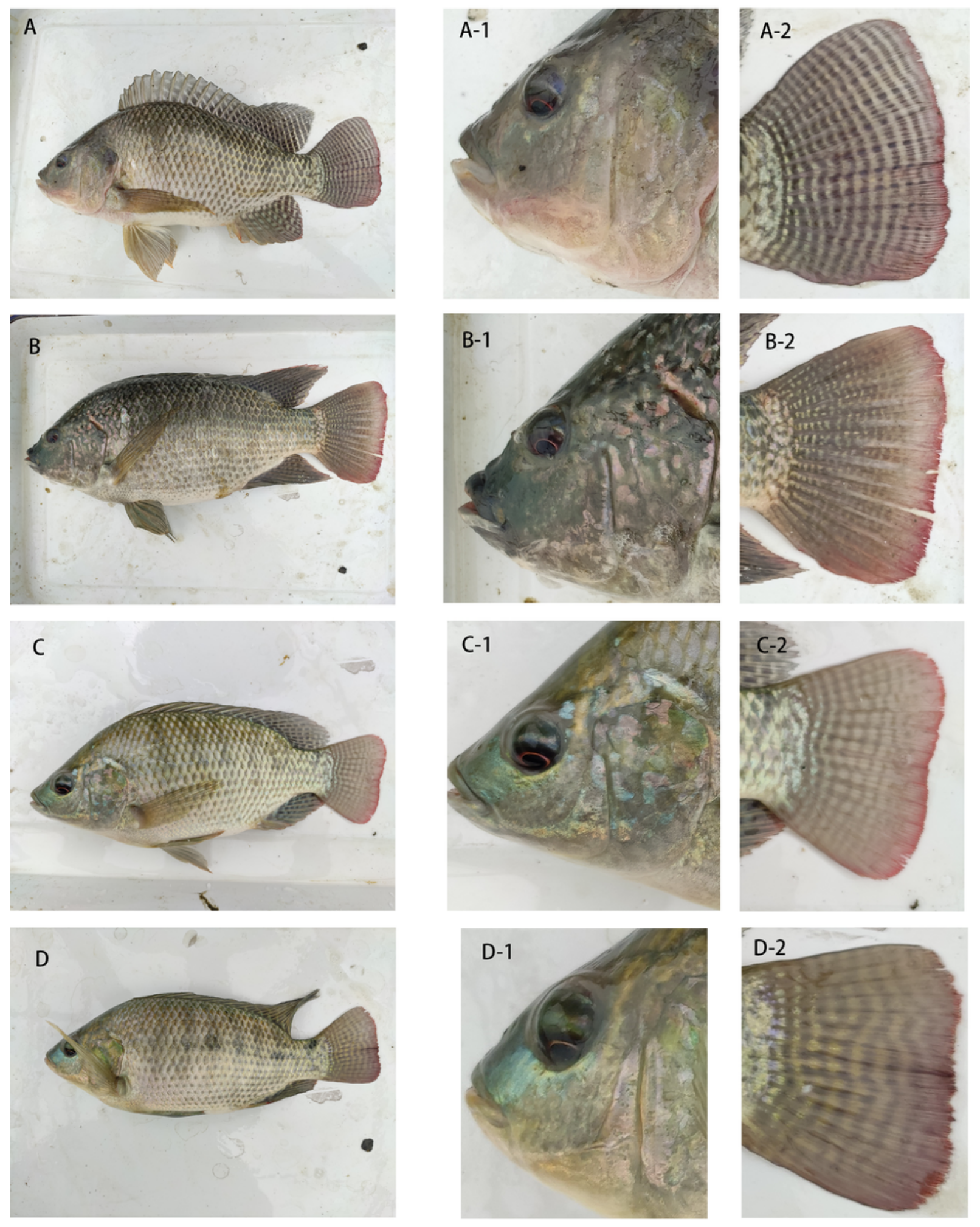

\section{Figure 1}

The image of four kinds of experimental-used tilapia with their operculum and caudal fin. Legends: $A$ : Oreochromis niloticus (N), A-1: Operculum of N, A-2: Caudal fin of N; B: Oreochromis aureus (A), B-1:

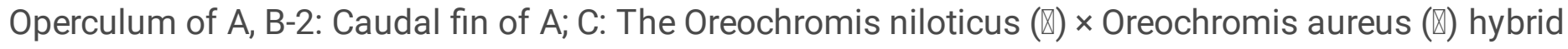
(AN), C-1: Operculum of AN, C-2: Caudal fin of AN; D: The Oreochromis niloticus $(\mathbb{D}) \times$ Oreochromis aureus (『) hybrid (NA), D-1: Operculum of NA, D-2: Caudal fin of NA. 


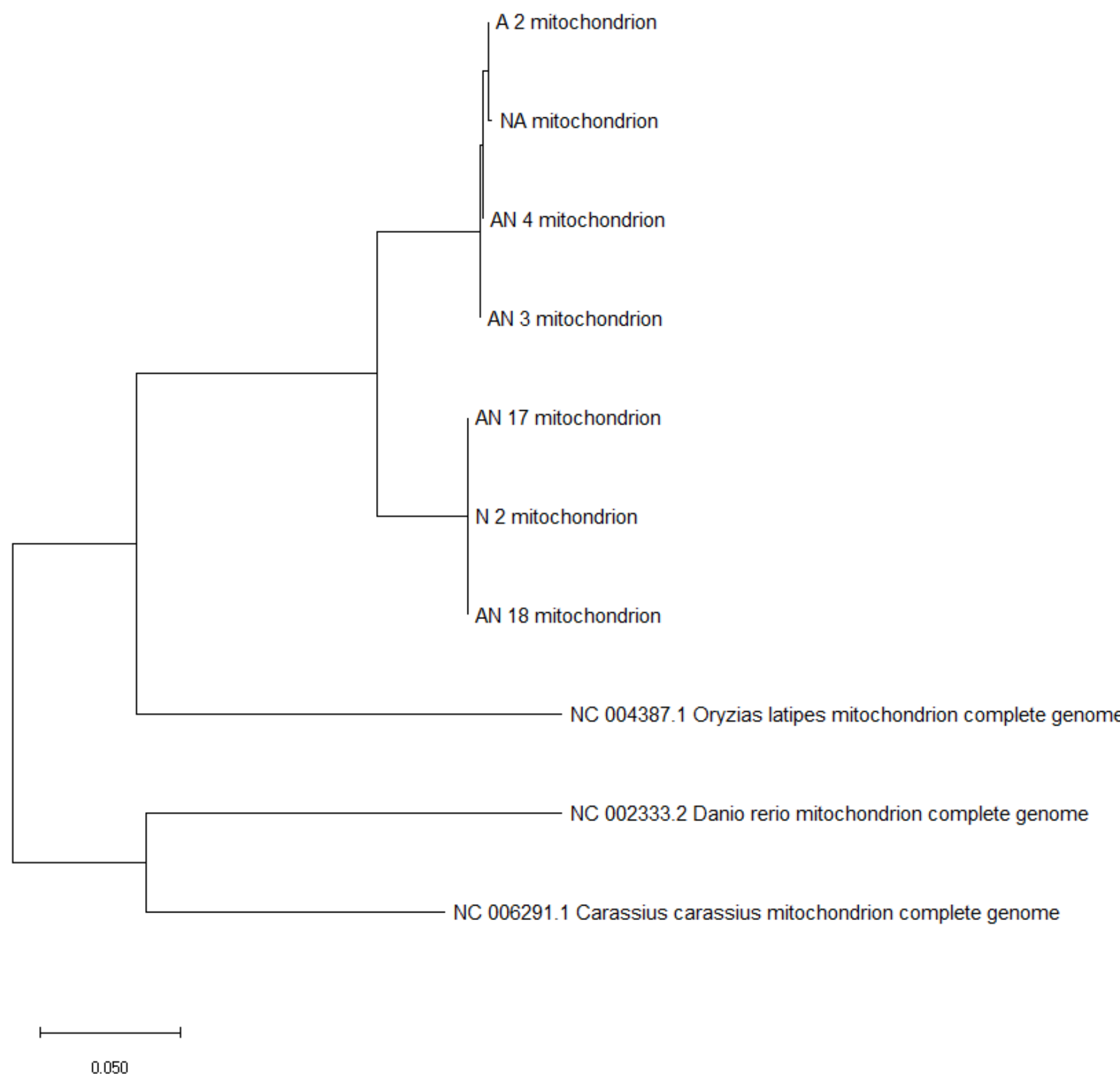

\section{Figure 2}

The phylogenetic tree of four kinds of experimental-used tilapia. Legends: A 2 Mitochondrion: The complete mt genome of Oreochromis aureus (A). N 2 Mitochondrion: The complete mt genome of Oreochromis niloticus (N). NA Mitochondrion: The complete mt genome of Oreochromis niloticus $(\mathbb{\nabla}) \times$

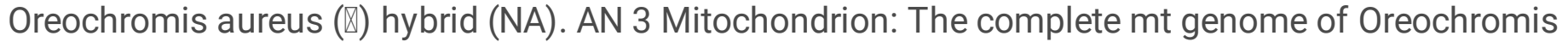

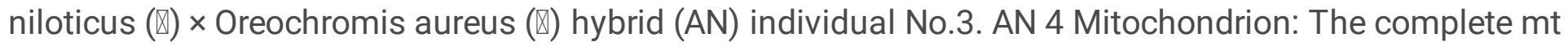

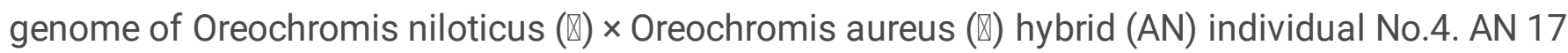

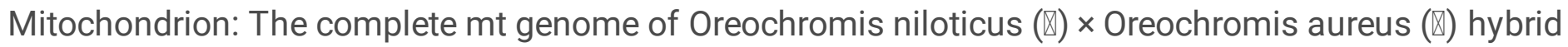


(AN) individual No.17. AN 18 Mitochondrion: The complete mt genome of Oreochromis niloticus $(\mathbb{\nabla}) \times$ Oreochromis aureus ( () hybrid (AN) individual No.18.

\section{Supplementary Files}

This is a list of supplementary files associated with this preprint. Click to download.

- Table1.xls

- Table2.xIs

- Table3.xls 